\title{
Cocoa Supplementation for Copper Deficiency Associated with Tube Feeding Nutrition
}

\author{
Yasuharu Tokuda ${ }^{1}$, Masayuki Kashima ${ }^{2}$, Mami Kayo ${ }^{2}$, \\ Nobuhiko Nakazato ${ }^{2}$ and Gerald H. Stein ${ }^{3}$
}

\begin{abstract}
Objective Because of an increasing number of case reports of copper deficiency associated with long-term tube feeding nutrition in Japan, we identified patients with copper deficiency associated with long-term tube feeding and described the prevalence, clinical data and cocoa treatment for these patients.

Materials and Methods We conducted a retrospective study to identify patients who were referred from long-term care institutions and had copper deficiency associated with tube feeding. We reviewed all serum copper concentration data during a 6-year period. We also compared admission and post-treatment peripheral blood counts.

Results Among 210 consecutive admissions with nutritional tube feeding from long-term care institutions $(\mathrm{N}=210)$, we identified 13 (6.2\%) patients with copper deficiency (aged 46-91; 12 women). All patients had anemia, while most had neutropenia. The range of serum copper concentrations of these patients was 0.1-2.4 $\mu \mathrm{g} / \mathrm{L}$ (normal; 6.8-12.8 $\mu \mathrm{g} / \mathrm{L}$ ). Their feeding formulas revealed a low copper content (5 to $12 \mu \mathrm{g}$ per $100 \mathrm{kcal}$ of each formula). Cocoa powder was used as the treatment. With cocoa supplements, the blood leukocyte count and hemoglobin significantly improved in all patients. Median leukocyte counts were $1,800 / \mathrm{mm}^{3}$ at ad-

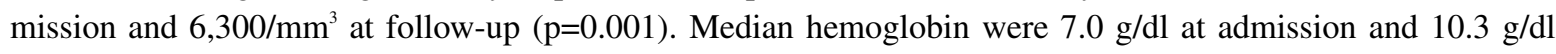
at follow-up $(\mathrm{p}=0.001)$. Two patients developed transient tachycardia as a possible adverse effect of cocoa. Conclusion We identified many cases with copper deficiency associated with tube feeding in Okinawa, Japan. Cocoa supplement appeared to be a safe and effective treatment. Increasing the copper content of Japanese tube feeding formulas should be considered for its prevention.
\end{abstract}

Key words: copper, enteral nutrition, anemia, leukopenia, cocoa

(DOI: 10.2169/internalmedicine.45.1525)

\section{Introduction}

Copper deficiency is relatively rare because of ubiquitous distribution of copper and its low daily requirements (1). In 1972, Karpel and Peden reported the first case of anemia and neutropenia due to copper deficiency in patients receiving total parenteral nutrition (2). In addition, recent sporadic case reports showed that patients receiving tube feeding as enteral nutrition can develop copper deficiency. The first such case was reported by Tamura et al in 1994 (3) and subsequent case reports followed thereafter exclusively from Ja- pan (3-5). Tamura et al suggested that the possible cause of these cases was the use of commercial formulations for tube feeding in Japan since they contained a low content of this essential trace element (3). We also reported a single case from our hospital (6). Since commercially available cocoa powder is rich in copper and is easily mixed into feeding formula, we and others previously suggested using cocoa supplementation for tube feeding formula to improve anemia and leukopenia in these patients $(6,7)$.

However, there are no studies to investigate the epidemiology and clinical characteristics of copper deficiency related to tube feeding in Japan. In addition, the available case

\footnotetext{
${ }^{1}$ Department of Medicine, St. Luke' International Hospita, Tokyo, ${ }^{2}$ Department of Medicine, Okinawa Chubu Hospital, Okinawa and ${ }^{3}$ Department of Medicine, College of Medicine, University of Florida, Gainesville, Florida, and University of Hawaii, Honolulu, Hawaii, USA Received for publication August 8, 2005; Accepted for publication June 19, 2006

Correspondence to Dr. Yasuharu Tokuda, Department of Medicine, St. Luke's International Hospital, and Center for Clinical Practice Research, St. Luke's Life Science Institute, 10-1 Akashi-cho, Chuo-ku, Tokyo 104-0044
} 
reports only described cocoa supplement as a treatment of copper deficiency. There are no formal evaluations for cocoa as an effective treatment of copper deficiency not only in clinical trials but also in retrospective observational studies. Thus, the objective of the present study was to analyze the prevalence of copper deficiency among tube feeding patients who were referred from long-term care institutions and to describe its clinical characteristics. We also examined the copper content of feeding formulas used for these patients and reported the safety and efficacy of cocoa supplementation.

\section{Patients and Methods}

We performed a single center, retrospective study during a six-year period (January 1998 -December 2003) to identify adult patients (20-year-old and older) on long-term tube feeding and low serum copper concentration. We reviewed the medical records of all patients on tube feeding who were referred from long-term care institutions and admitted to Okinawa Chubu Hospital, a 550-bed community general hospital in Okinawa, Japan. We also reviewed all data of serum copper concentration tests performed in this patient population.

The present case inclusion criteria were the following 2 conditions; 1) patients who received tube feeding before admission as their sole nutritional route for at least a 2-month duration; and 2) patients who had a serum copper concentration of less than $6.8 \mu \mathrm{g} / \mathrm{L}$ (normal range; 6.8-12.8 $\mu \mathrm{g} / \mathrm{L}$ ). We examined the medical records specifically for signs and symptoms of copper deficiency such as myelopathy and hematological abnormality $(8,9)$. We also reviewed the past medical history of the patients to identify the possibility of Menkes disease, a phenotype from ATP-7A gene mutations (i.e. peculiar hair, failure to thrive, mental retardation, and severe cerebellar neurodegeneration) or an occipital horn syndrome, a milder phenotype from ATP-7A gene mutations (i.e. connective-tissue abnormalities) $(10,11)$. We also analyzed the peripheral leukocyte count with differentials, blood hemoglobin concentrations, and platelet counts in these patients. The serum copper concentration was measured by gas chromatography in the SRL Corporation (Special Reference Laboratory), Tokyo, Japan.

Since anemia and/or neutropenia in these patients could also be caused by other conditions such as iron deficiency, we also collected data for serum concentrations of iron, total iron binding capacity (TIBC), and transferrin saturation, ferritin, vitamin B12, and folic acid to rule out these causes. In addition, we searched for the presence of gastrointestinal bleeding in patients with copper deficiency as a potential cause of anemia. For primary hematological diseases such as aplastic anemia, multiple myeloma and malignant lymphoma as potential causes of anemia and/or neutropenia, we reviewed the bone marrow examinations performed in patients with copper deficiency to rule out these diseases. Since myelodysplastic changes are a typical picture of copper defi-
Table 1. Clinical Characteristics of Patients with Copper Deficiency

\begin{tabular}{cccccc}
\hline No. & Age $(\mathrm{y})$ & Gender & Disease & TFD & feeding formula \\
\hline 1 & 71 & $f$ & stroke & 7 & N/A \\
2 & 83 & $f$ & NMD & 5 & TERUMIRU \\
3 & 46 & $f$ & stroke & 108 & ISOCAL \\
4 & 70 & $\mathrm{~m}$ & stroke & 36 & N/A \\
5 & 90 & $\mathrm{f}$ & AD & N/A & N/A \\
6 & 67 & $\mathrm{f}$ & stroke & 24 & N/A \\
7 & 82 & $\mathrm{f}$ & RA & 6 & MA-2 \\
8 & 69 & $\mathrm{f}$ & stroke & 12 & ISOCAL \\
9 & 82 & $\mathrm{f}$ & NPH & 6 & ISOCAL \\
10 & 91 & $\mathrm{f}$ & stroke & 12 & L5 \\
11 & 91 & $\mathrm{f}$ & AD & 2 & N/A \\
12 & 66 & $\mathrm{f}$ & RA & 4 & ISOCAL \\
13 & 71 & $\mathrm{f}$ & NMD & 96 & N/A \\
\hline
\end{tabular}

${ }^{*}$ Commercial names are provided for feeding formulas

TFD, tube feeding duration (months); NMD, neuromuscular disease; AD, Alzheimer's disease RA, rheumatoid arthritis; NPH, normal pressure hydrocephalus; N/A, data not available.

ciency in bone marrow examination (12), these findings were regarded as supportive evidence for copper deficiency as a cause of anemia and neutropenia.

We collected data about the treatment in patients with copper deficiency. Since our institution previously recommended the empiric treatment with commercially available cocoa powder for copper deficiency, cocoa was the treatment of choice during the study period. The commercially available cocoa powder contained an average of elemental copper of $3.8 \mathrm{mg} / 100 \mathrm{~g}$ weight. Most patients were prescribed $10 \mathrm{~g}$ of cocoa (total copper content $0.38 \mathrm{mg}$ ) as the initial loading dose for about 2 weeks based on the recommendation of the previous reports. The maintenance dose was $5 \mathrm{~g}$ of cocoa (total copper content $0.19 \mathrm{mg}$ ), as described in previous case reports $(6,7)$. A slight modification for the dosing schedule was employed based on approximate individual body size during the maintenance period.

We reviewed the medical records without a specified schedule during the cocoa supplementation period. We also collected data of complete blood count during this period. We defined the follow-up data as complete blood count data after two or three month intervals from initiating cocoa supplements. The study received approval from the Institutional Review Board of Okinawa Chubu Hospital.

We determined the prevalence of copper deficiency in all tube-fed patients admitted to our hospital from long-term care institutions during the 6-year period. Exact binominal distributions were used for calculating its $95 \%$ confidence interval (CI). We calculated Spearman correlation coefficients and its p-values to determine whether or not there were correlations between serum copper concentrations and leukocyte counts or neutrophil counts. Since sample size of patients with copper deficiency was small $(n=14)$ and blood leukocyte counts and hemoglobin concentrations were not normally distributed, we used non-parametric test (Wilcoxon signed rank test) as single-group comparisons between admission and follow-up data. Median values were calculated accordingly. A two-tailed p-value cutoff of 0.05 was used as statistical significance. The SPSS statistical package (version 12.0J) was used for all analyses (SPSS Japan Inc., Tokyo, Japan). 
Table 2. Laboratory Data of Patients with Copper Deficiency Associated with Tube Feeding

\begin{tabular}{ccccccccccc}
\hline No. & Leukocyte, $/ \mu \mathrm{L}$ & Neutrophil, $/ \mu \mathrm{L}(\%)$ & Lymphocyte, $/ \mu \mathrm{L}(\%)$ & $\mathrm{Hb}, \mathrm{g} / \mathrm{dL}$ & $\mathrm{MCV}, \mathrm{fL}$ & $\mathrm{PLT}, / \mu \mathrm{L}$ & $\mathrm{Cu}, \mu \mathrm{g} / \mathrm{L}$ & $\mathrm{TIBC}, \mathrm{mg} / \mathrm{dl}$ & $\mathrm{Fe}, \mathrm{mg} / \mathrm{dl}$ & $\mathrm{TS}, \%$ \\
\hline 1 & 1,500 & $285(19)$ & $765(51)$ & 4.8 & 97 & 389,000 & 0.7 & 296 & 13 & 4.39 \\
2 & 6,100 & $3,355(55)$ & $793(13)$ & 5.3 & 95 & 150,000 & 1.7 & 190 & 40 & 21.05 \\
3 & 2,300 & $690(30)$ & $460(20)$ & 7.6 & 111 & 184,000 & 0.4 & 187 & 17 & 9.09 \\
4 & 600 & $24(4)$ & $492(82)$ & 4.0 & 110 & 122,000 & 0.1 & 136 & 9 & 6.62 \\
5 & 1,600 & $\mathrm{~N} / \mathrm{A}$ & $848(53)$ & 9.0 & 116 & 161,000 & 0.6 & $\mathrm{~N} / \mathrm{A}$ & $\mathrm{N} / \mathrm{A}$ & $\mathrm{N} / \mathrm{A}$ \\
6 & 3,900 & $2,495(64)$ & $819(21)$ & 7.0 & 103 & 258,000 & 0.3 & 216 & 25 & 11.57 \\
7 & 1,900 & $\mathrm{~N} / \mathrm{A}$ & $\mathrm{N} / \mathrm{A}$ & 7.1 & 103 & 125,000 & 2.4 & 173 & 49 & 28.32 \\
8 & 1,600 & $144(9)$ & $784(49)$ & 5.2 & 104 & 296,000 & 0.2 & $\mathrm{~N} / \mathrm{A}$ & $\mathrm{N} / \mathrm{A}$ & $\mathrm{N} / \mathrm{A}$ \\
9 & 1,800 & $\mathrm{~N} / \mathrm{A}$ & $\mathrm{N} / \mathrm{A}$ & 5.3 & 134 & 242,000 & 0.3 & $\mathrm{~N} / \mathrm{A}$ & $\mathrm{N} / \mathrm{A}$ & $\mathrm{N} / \mathrm{A}$ \\
10 & 3,200 & $288(9)$ & $1,440(45)$ & 8.0 & 99 & 240,000 & 0.7 & $\mathrm{~N} / \mathrm{A}$ & $\mathrm{N} / \mathrm{A}$ & $\mathrm{N} / \mathrm{A}$ \\
11 & 1,600 & $640(40)$ & $448(28)$ & 8.3 & 110 & 108,000 & 0.5 & $\mathrm{~N} / \mathrm{A}$ & $\mathrm{N} / \mathrm{A}$ & $\mathrm{N} / \mathrm{A}$ \\
12 & 1,200 & $432(36)$ & $540(45)$ & 8.7 & 96 & 164,000 & 0.6 & $\mathrm{~N} / \mathrm{A}$ & $\mathrm{N} / \mathrm{A}$ & $\mathrm{N} / \mathrm{A}$ \\
13 & 1,900 & $456(24)$ & $1,102(58)$ & 6.5 & 114 & 347,000 & 0.4 & 250 & 19 & 7.60 \\
\hline
\end{tabular}

$\mathrm{Hb}$, blood hemoglobin; MCV, mean corpuscular volume; PLT, blood platelet; $\mathrm{Cu}$, serum copper

TIBC, total iron binding capacity; $\mathrm{Fe}$, serum iron; TS, transferrin saturation; N/A, data not available.

Results

We identified 13 patients meeting our inclusion criteria. There were 12 women aged 46-91 years old and 1 man aged 70 years old. These 13 patients comprised $6.2 \%(95 \%$ CI, 3.3-10.4\%) among consecutively admitted patients with nutritional tube feeding from long-term care institutions $(\mathrm{N}=$ 210). Table 1 shows the clinical characteristics. Of 13 patients, 9 received their tube feeding through naso-gastric feeding tubes and 4 had percutaneous endoscopic gastrostomy feeding tubes. In terms of baseline diseases for tube feeding, 6 had cerebrovascular disease, 2 had a neuromuscular disease, 2 had Alzheimer's disease, 2 had rheumatoid arthritis, and 1 normal pressure hydrocephalus. There were no patients who had clinical features compatible with Menkes disease or occipital horn syndrome based on a review of past medical history. The patients had received tube feeding for a median duration of 7 months (range, 2-108 months). All were bedridden with mild to severe extremity contractures. No signs or symptoms consistent with copper deficiency were noted in the medical records of these patients. There were no patients with clinical suspicion for myelopathy and no patients underwent studies for myelopathy including magnetic resonance imaging and somatosensory evoked potential analysis.

Table 2 shows the laboratory data at admission. All patients had low serum copper concentrations with a median of $0.5 \mu \mathrm{g} / \mathrm{L}$ (range; 0.1-2.4 $\mu \mathrm{g} / \mathrm{L}$ ). Because serum copper measurement is a relatively expensive test, most patients had this test only for baseline copper concentrations. Median serum albumin was $0.26 \mathrm{~g} / \mathrm{L}$ (range; 0.23-0.33 g/L). Five patients received red blood cell transfusions based on clinical indication. Bone marrow examinations were performed in 2 patients, and both showed myelodysplastic-like histology which was compatible with copper deficiency. There were 4 patients with serum transferrin saturation of $10 \%$ or lower among 7 patients for whom these data were available. Since the iron content in the feeding formula for our patients was not considered to be low and we considered copper deficiency as a primary factor for leucopenia and anemia in our patients, there were no patients who received additional iron supplementation treatment in addition to cocoa treatment.

Most patients with copper deficiency showed hematological abnormalities except for platelet counts. All 13 patients had macrocytic anemia; the range of hemoglobin was 4.0$9.0 \mathrm{~g} / \mathrm{dL}$ (median; $7.0 \mathrm{~g} / \mathrm{dL}$ ) and the range of mean corpuscular volume was 95-134 fL (median; $104 \mathrm{fL}$ ). The range of leukocyte count was $600-6,100 / \mu \mathrm{L} ; 3$ patients had leukopenia with a total leukocyte count of $1,500 / \mu \mathrm{L}$ or lower. Although 3 patients had missing data for leukocyte differential tests, the range of neutrophil counts in 10 patients was 24 to 3,355/ $\mu \mathrm{L} ; 6$ patients showed neutropenia (neutrophil count of $500 / \mu \mathrm{L}$ or lower). The range of platelet counts of all patients with copper deficiency was 108,000 to $389,000 / \mu \mathrm{L}$ (median; 184,000/ $\mu \mathrm{L}$ ).

There were 2 patients with outlying values (more than $75 \%$ percentile) of their leukocyte counts. One patient was an 83-year-old woman with neuromuscular disease on tube feeding for 5 months. Her tube feeding formula was TERUMIRU $^{\circledast}$ (copper content $10 \mu \mathrm{g} / 100 \mathrm{kcal}$; carbohydrate type, dextrins). Her blood test showed leukocyte count of $6,100 / \mu \mathrm{L}$ (neutrophil count; 3,355/ $/ \mathrm{L}$ ), hemoglobin $5.3 \mathrm{~g} /$ $\mathrm{dL}$, platelets $150,000 / \mu \mathrm{L}$ and copper concentration of 1.7 $\mu \mathrm{g} / \mathrm{L}$. The other was a 67 -year-old woman who had a stroke and was on tube feeding for 24 months. The information of her tube feeding formula was not available. Her blood test showed a leukocyte count of $3,900 / \mu \mathrm{L}$ (neutrophil count; $2,495 / \mu \mathrm{L}$ ), hemoglobin $7.0 \mathrm{~g} / \mathrm{dL}$, platelets $258,000 / \mu \mathrm{L}$ and copper concentration of $0.3 \mu \mathrm{g} / \mathrm{L}$.

There was no statistically significant correlation between blood leukocyte counts and serum copper concentrations in patients with copper deficiency, although a positive correlation was noted as a trend (Spearman correlation coefficient= $0.280, \mathrm{p}=0.355$ ). Likewise, there was no statistically significant correlation between blood neutrophil counts and serum copper concentrations. However, we noted a greater positive correlation trend (Spearman correlation coefficient $=0.354$, $\mathrm{p}=0.316$ ). Between blood lymphocyte counts and serum copper concentrations, there was no statistically significant correlation (Spearman correlation coefficient $=0.270, \mathrm{p}=0.422$ ).

Our review identified 2 patients with copper deficiency and chronic inflammatory disease. In these 2 patients who had rheumatoid arthritis, one patient (82-year-old woman) 
Table 3. Trace Element Vitamin Composition and Carbohydrate Type of Defined-fomula Diet Used for Patients

\begin{tabular}{|c|c|c|c|c|c|}
\hline Constituents $^{*}$ & TERUMIRU ${ }^{\circledR}$ & ISOCAL $^{\circledR}$ & MA-2 ${ }^{\circledR}$ & $\mathrm{L} 5^{\circledR}$ & RDA† \\
\hline Copper $(\mu \mathrm{g})$ & 10 & 12 & 5 & 5 & 52 \\
\hline Iron (mg) & 0.85 & 0.9 & 1 & 1.2 & 0.37 \\
\hline Manganase $(\mu \mathrm{g})$ & 30 & 20 & 2.5 & 10 & N/D \\
\hline Zinc (mg) & 0.4 & 0.16 & 0.3 & 0.32 & 0.52 \\
\hline Vitamin A (IU) & 250 & 250 & 300 & 150 & 133 \\
\hline Vitamin B1 (mg) & 0.125 & 0.19 & 0.12 & 0.16 & 0.06 \\
\hline Vitamin B2 (mg) & 0.17 & 0.22 & 0.18 & 0.22 & 0.07 \\
\hline Vitamin B6 (mg) & 0.25 & 0.25 & 0.3 & 0.24 & 0.09 \\
\hline Vitamin B12 $(\mu \mathrm{g})$ & 0.625 & N/A & 0.3 & 0.45 & 0.18 \\
\hline Vitamin C (mg) & 30 & 15 & 10 & 13 & 7.4 \\
\hline Vitamin D (IU) & 12.5 & 20 & 20 & 8 & N/D \\
\hline Vitamin E (mg) & 2.7 & 3.4 & 1.8 & 0.6 & N/D \\
\hline Niacin (mg) & 2 & 2.5 & 2 & 2.5 & 0.67 \\
\hline Folate $(\mu \mathrm{g})$ & 25 & 20 & 30 & 32 & 18 \\
\hline Pantothenic acid (mg) & 0.9 & 1.25 & 1 & 1 & N/D \\
\hline Carbohydrate type & Starch (Dextrins) & $\begin{array}{l}\text { Starch (Dextrins) } \\
\text { Sucrose }\end{array}$ & Starch (Dextrins) & $\begin{array}{l}\text { Starch (Dextrins) } \\
\text { Sucrose }\end{array}$ & \\
\hline
\end{tabular}

*Amount included in $100 \mathrm{kcal}$ of the defined-formula diet.

†Modified from Recommended dietary allowance in $100 \mathrm{kcal}$ for women of 70 years old and older with a low daily activity

by Ministry of Health, Welfare and Labor, Tokyo, Japan, 2005

(http://www.mhlw.go.jp/houdou/2004/11/h1122-2a.html)

$\mathrm{N} / \mathrm{D}$, data not determined; N/A, data not available

had a serum copper concentration of $2.4 \mu \mathrm{g} / \mathrm{L}$ and leukocyte counts of $1,900 / \mu \mathrm{L}$ (missing data for neutrophil count). The other (66-year-old woman) showed a serum copper concentration of $0.6 \mu \mathrm{g} / \mathrm{L}$ and leukocyte counts of $1,200 / \mu \mathrm{L}$ (neutrophil count; $432 / \mu \mathrm{L}$ ).

Based on the product information of each commercialdefined formula, Table 3 shows the trace elements and vitamins. The typical commercial tube feeding formula per 100 kcal contained 12.3-15.0 g of carbohydrate, 3.3-5.0 g of protein, 2.2-4.2 g of lipids, electrolytes, trace elements, and vitamins. The patients received the formulas with a range of 900-1,500 kcal/day. Those tube feeding formulas contained 5-12 $\mu \mathrm{g}$ of copper per $100 \mathrm{kcal}$. Thus, total daily copper intake was about $50-180 \mu \mathrm{g}$ based on $900-1,500 \mathrm{kcal} /$ day feeding. This estimation of copper intake was lower than the recommended daily requirement issued by the Japanese government (700-800 $\mu \mathrm{g}$ copper/day) (13). In terms of the type of carbohydrate in the formulas, 2 formulas (TERUMIRU ${ }^{\circledR}$ and MA- $2^{\circledR}$ ) used only dextrins (consisting of glucose), and 2 formulas (ISOCAL ${ }^{\circledR}$ and $\mathrm{L}^{\circledR}{ }^{\circledR}$ ) used dextrins and sucrose (consisting of glucose and fructose). We were unable to specify the exact formulas of the tube feedings in 7 of 14 patients, since their medical records omitted this information.

During the therapeutic supplementation of cocoa powder to tube feedings, anemia and leukopenia significantly improved at follow-up. Since leukocyte differentials tests were not performed in most patients at follow-up, we used leukocyte count data as surrogate markers for estimating treatment effectiveness. Fig. 1 plots change in blood leukocyte count of individual patients at admission (baseline) and follow-up test after 2 to 3 months of cocoa administration. Median blood leukocyte count was $1,800 / \mathrm{mm}^{3}$ at admission and $6,300 / \mathrm{mm}^{3}$ at follow-up; Wilcoxon signed rank test

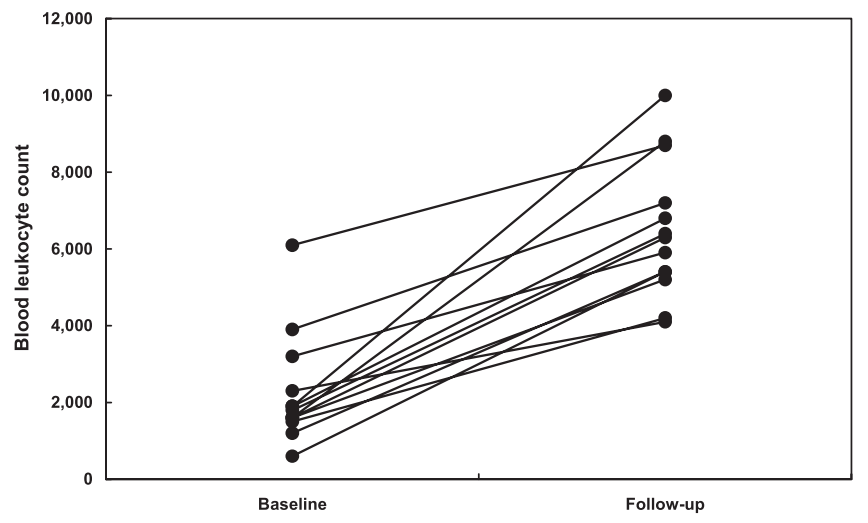

Figure 1. Blood leukocyte count (per $\mathrm{mm}^{3}$ ) before and after cocoa supplementation at follow-up 2-3 months later.

showed a significant increase in blood leukocyte $(\mathrm{p}=0.001)$. Fig. 2 shows the change in blood hemoglobin of individual patients at admission and follow-up. Median blood hemoglobin was $7.0 \mathrm{~g} / \mathrm{dL}$ at admission and $10.3 \mathrm{~g} / \mathrm{dL}$ at follow-up; Wilcoxon signed rank test also showed a significant increase in blood hemoglobin ( $\mathrm{p}=0.001)$.

Based on our review of the medical records, anemia and neutropenia had not recurred in any patient after introducing cocoa supplementation. We recognized transient tachycardia as a possible side effect of cocoa supplements in 2 patients (maximum heart rate 90 and130 per minute, respectively). Tachycardia disappeared soon after reducing the dose of cocoa powder. No patient experienced any other significant side effects. One patient died from nosocomial pneumonia during hospitalization. The medical record of this patient showed that it was unrelated to cocoa supplementation. 


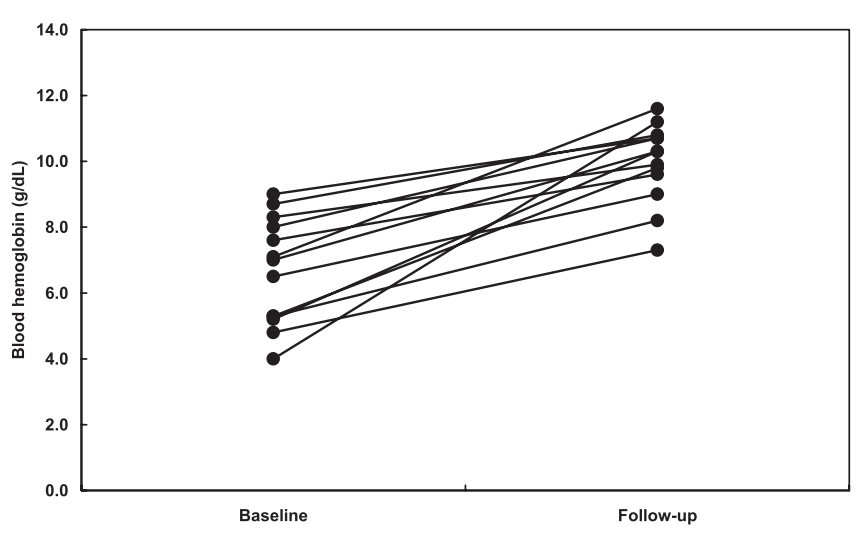

Figure 2. Blood hemoglobin before and after cocoa supplementation at follow-up 2-3 months later.

\section{Discussion}

Patients on nutritional enteral tube feeding may develop anemia and leukopenia from copper deficiency. Our six-year retrospective study identified 13 patients with a low serum copper concentration. An analysis of the feeding formulas used in these patients showed that the copper content per serving of the formulas was low compared to the recommended daily allowance. All patients showed macrocytic anemia; some patients had neutropenia. There was improvement of blood leukocyte count and hemoglobin using copper-rich cocoa powder supplementation. This can also be supportive evidence for copper deficiency as etiology of their anemia and/or neutropenia.

The present data showed that there were at least six percent cases of copper deficiency among the admitted patients on tube feeding. Since serum copper was measured only for patients who were clinically suspected to have copper deficiency, the true prevalence could be higher than our estimation. However, this magnitude of prevalence does not appear small when considering the huge number of patients receiving tube feeding. Anemia and/or neutropenia with unrecognized copper deficiency may lead to clinical events including admission to acute care hospitals, expensive evaluations for anemia and neutropenia, and potential multiple blood transfusions. Further, copper deficiency may lead to reduced immune function including neutrophil function, lymphocyte function and humoral immunity. Increased morbidity was suggested in patients with copper deficiency (14). In addition, copper deficiency can cause myelopathy (9), and inflammatory lung disease by neutrophil-endothelial interactions (15); it also may impair cardiovascular health $(16,17)$. Since most patients in our study were bedridden for long term duration with severe difficulty in communication and mobilization, they could not present symptoms and signs of clinical myelopathy associated with copper deficiency; we did not perform magnetic resonance imaging and somatosensory evoked potential analysis to identify occult myelo- pathy (9). Thus, myelopathy may have occurred in our study patients.

There is a slight variation in the daily nutritional requirements for copper intake among governments. The recommended daily allowance of copper by the US National Academy of Sciences is $900 \mu \mathrm{g}$ per day for adult men and women (18). Japanese government recommends a dietary allowance of $700 \mu \mathrm{g}$ per day in women and $800 \mu \mathrm{g}$ per day in men aged 18 years old and older (13).

Although it is unclear why gender distribution of our patients showed more women than men (12 versus 1$)$, there are possible explanations for this gender difference. First, longevity is greater for women compared to men in Japan; many more women can stay in long-term care institutions than men. Therefore, we may receive more women patients with copper deficiency from those institutions. The second reason may be that women could be more vulnerable to copper deficiency from multiple mechanisms including the possibility of reduced intestinal copper absorption. However, this hypothesis has not been investigated.

The product information of feeding formulas used for our patients revealed the low content of copper per $100 \mathrm{kcal}$ in comparison to the recommended daily allowance. Also considering the increased case reports of copper deficiency in Japan, the widespread use of the available feeding formulas was likely to be the cause (3). In the recently available feeding formulas of Japan, the copper content per serving was small compared to the aforementioned daily requirement; there was little free copper. This was also compatible with the fact that recent cases with tube feeding-associated copper deficiency were reported exclusively from Japan (6). In addition, copper-rich natural products were used previously until defined-formula became commercially available. During this period, there were few reports of copper deficiency associated with tube feeding. Therefore, we recommend that the sole use of a defined-formula containing smaller amounts of copper should be avoided especially for long-term use. When defined formulas are used, copper supplements such as cocoa should be added to feeding formulas to restore normal blood copper levels.

Our study showed that cocoa powder is an effective copper supplement in this setting. Cocoa can be produced during cacao fermentation and roasting; cocoa and chocolate can be processed together (6). It is relatively inexpensive and easy to mix with the conventional feeding formula. In the absence of difficult to conduct controlled studies we may propose that the government require increased copper content in tube feeding formulas. We noted transient tachycardia as possible adverse effects of cocoa supplements. This was probably caused by the caffeine-like substance contained in cocoa powder (6). To prevent tachycardia and potential tachyarrhythmia from using cocoa powder, we recommend initiating small dose of cocoa and regularly monitoring heart rate and blood pressure especially during the initial treatment with cocoa supplements. There are also other foods containing high amounts of copper such as seaweed, 
oyster and beans. However, cocoa is convenient for its ease of adding to feeding formula.

There were several conditions associated with copper deficiency including case reports of patients receiving total parenteral nutrition (19-23), those with short bowel syndrome $(24,25)$, and those with partial gastrectomy $(12,26$, $27)$. In addition, sporadic case reports also suggested that inadequate diet alone or genetic disorders affecting proteins of copper metabolism could cause copper deficiency (28-30), including Menkes disease and occipital horn syndrome (10). Although the medical record review of our patients did not reveal the past clinical history suggestive for Menkes disease and occipital horn syndrome, we could not eliminate the possibility of genetic disorders involving proteins of copper metabolism (10). Malnourished elderly patients may develop a defect of intestinal copper absorption. This process could contribute to copper deficiency in our patients who were mostly elderly (26). In addition, fructose feeding could also be contributing factor for copper deficiency syndrome in 2 patients of our study (31), since the fructose feeding could contribute to altered copper metabolism. Furthermore, zinc is known to have a competitive effect on copper during absorption (32). Since serum zinc was not measured in our patients, we could not estimate the contribution of zinc to copper concentrations in our patients.

The clinical course of our patients is similar to a case report of acquired copper deficiency following prolonged jejunostomy feeding (33). Their patient developed macrocytic anemia and leucopenia after receiving long-term enteral nutrition. However, copper deficiency in their case was not resolved by copper supplementation via jejunostomy feeding. In contrast, in all of our patients anemia and leucopenia improved by cocoa supplementation via gastrostomy tube. In fact, the authors suggested that oral or gastrostomy tube route was preferred for restoring copper deficiency (33).

Various diseases and conditions can cause anemia and/or leucopenia in patients on tube feeding. Because most patients were elderly and malnourished in our study, their anemia and leucopenia could have been related to other nutri- tional deficiency, overall malnutrition, or primary bone marrow disorders. We performed bone marrow examination in only 2 patients to rule out hematologic disorders. However, all patients identified by our diagnostic criteria had a low serum copper concentration. In addition, hematological improvement after copper-rich cocoa supplementation supports copper deficiency as the most likely cause of their anemia and/or neutropenia. On the other hand, iron deficiency can accompany copper deficiency states; there were patients with low serum transferrin saturation in a report (34). Although iron supplementation was not prescribed for our patients, iron deficiency might be a contributory factor for anemia in our patients.

Because of our retrospective design, our calculation of the prevalence of copper deficiency may be a subject to underestimation. Measurements of serum copper concentrations were not performed for all admitted patients on tube feeding in our hospital, since the majority of them had no clinical indication to suspect copper deficiency. However, normal values of hemoglobin and leukocyte count cannot exclude subclinical copper deficiency. Furthermore, we analyzed only patients who were referred to our hospital from longterm care institutions. The actual prevalence for copper deficiency in patients of these institutions may be much greater.

In conclusion, we uncovered 13 cases of copper deficiency among patients receiving defined tube feeding formulas. Copper deficiency should be considered in the differential diagnosis of patients on long-term tube feeding with otherwise unexplained anemia and/or leucopenia. We successfully treated their hematologic abnormalities by adding cocoa powder to their tube feeding formulas. Most importantly, we recommend increasing the copper content of all defined formulas or the use of copper-enriched formulas for preventing its deficiency in Japan.

We thank Dr. Jun Miyara and Dr. Kouji Kubota for their supports for our study. We also thank Ms. Tomoko Yonaha for her excellent secretarial work.

\section{References}

1. Uauy R, Olivares M, Gonzalez M. Essentiality of copper in humans. Am J Clin Nutr 67 (5 Suppl): 952S-959S, 1998.

2. Karpel JT, Peden VH. Copper deficiency in long-term parenteral nutrition. J Pediatr 80: 32-36, 1972.

3. Tamura $\mathrm{H}$, Hirose $\mathrm{S}$, Watanabe $\mathrm{O}$, et al. Anemia and neutropenia due to copper deficiency in enteral nutrition. JPEN J Parenter Enteral Nutr 18: 185-189, 1994.

4. Masugi J, Amano M, Fukuda T. Copper deficiency anemia and prolonged enteral feeding. Ann Intern Med 121: 386, 1994.

5. Banno S, Niita M, Kikuchi M, et al. Anemia and neutropenia in elderly patients caused by copper deficiency for long-term enteral nutrition. Rinsho Ketsueki 35: 1276-1281, 1994 (in Japanese).

6. Kato J, Toyozato N, Hattori T, Motomura K, Tokuda Y. Long-term enteral nutrition-associated copper deficiency anemia and leukopenia: A case report and review of the literature. Journal of Okinawa Chubu Hospital 24: 20-23, 1998.
7. Wakugami K, Suenaga H, Egashira A, et al. Copper supplement with cocoa for copper deficiency in patients with long-term enteral nutrition. Nippon Ronen Igakkai Zasshi 37: 304-308, 2000 (in Japanese).

8. Fleming CR. Trace element metabolism in adult patients requiring total parenteral nutrition. Am J Clin Nutr 49: 573-579, 1989.

9. Kumar N, Gross JB Jr, Ahlskog JE. Copper deficiency myelopathy produces a clinical picture like subacute combined degeneration. Neurology 63: 33-39, 2004.

10. Miyajima H. Genetic disorders affecting proteins of iron and copper metabolism: clinical implications. Intern Med 41: 762-769, 2002.

11. Tapiero H, Townsend DM, Tew KD. Trace elements in human physiology and pathology. Copper. Biomed Pharmacother 57: 386398, 2003.

12. Gregg XT, Reddy V, Prchal JT. Copper deficiency masquerading 
as myelodysplastic syndrome. Blood 100: 1493-1495, 2002.

13. Ministry of Health, Labor and Welfare. Recommended Dietary Allowance for Japanese Nutrition. http://www.mhlw.go.jp/houdou/ 2004/11/h1122-2a.html.

14. Bonham M, O'Connor JM, Hannigan BM, Strain JJ. The immune system as a physiological indicator of marginal copper status? $\mathrm{Br}$ J Nutr 87: 393-403, 2002.

15. Lominadze D, Saari JT, Percival SS, Schuschke DA. Proinflammatory effects of copper deficiency on neutrophils and lung endothelial cells. Immunol Cell Biol 82: 231-238, 2004.

16. Schuschke DA. Dietary copper in the physiology of the microcirculation. J Nutr 127: 2274-2281, 1997.

17. Saari JT, Schuschke DA. Cardiovascular effects of dietary copper deficiency. Biofactors 10: 359-375, 1999.

18. Food and Nutrition Board IoM. Dietary Reference Intakes for Vitamin A, Vitamin K, Arsenic, Boron, Chromium, Copper, Iodine, Iron, Manganese, Molybdenum, Nickel, Silicon, Vanadium, and Zinc Washington, DC, The National Academies Press, 2000.

19. Sriram K, O'Gara JA, Strunk JR, Peterson JK. Neutropenia due to copper deficiency in total parenteral nutrition. JPEN J Parenter Enteral Nutr 10: 530-532, 1986.

20. Fujita M, Itakura T, Takagi Y, Okada A. Copper deficiency during total parenteral nutrition: clinical analysis of three cases. JPEN J Parenter Enteral Nutr 13: 421-425, 1989.

21. Takeuchi M, Tada A, Yoshimoto S, Takahashi K. Anemia and neutropenia due to copper deficiency during long-term total parenteral nutrition. Rinsho Ketsueki 34: 171-176, 1993 (in Japanese).

22. Wasa M, Satani M, Tanano H, Nezu R, Takagi Y, Okada A. Copper deficiency with pancytopenia during total parenteral nutrition. JPEN J Parenter Enteral Nutr 18: 190-192, 1994.

23. Fuhrman MP, Herrmann V, Masidonski P, Eby C. Pancytopenia af- ter removal of copper from total parenteral nutrition. JPEN J Parenter Enteral Nutr 24: 361-366, 2000.

24. Zidar BL, Shadduck RK, Zeigler Z, Winkelstein A. Observations on the anemia and neutropenia of human copper deficiency. Am J Hematol 3: 177-185, 1977.

25. Spiegel JE, Willenbucher RF. Rapid development of severe copper deficiency in a patient with Crohn's disease receiving parenteral nutrition. JPEN J Parenter Enteral Nutr 23: 169-172, 1999.

26. Hayton BA, Broome HE, Lilenbaum RC. Copper deficiencyinduced anemia and neutropenia secondary to intestinal malabsorption. Am J Hematol 48: 45-47, 1995.

27. Toyama T, Kubuki Y, Suzuki M, Tsubouchi H. Copper deficiency anemia and neutropenia secondary to total gastrectomy. Rinsho Ketsueki 41: 441-443, 2000 (in Japanese).

28. Williams DM. Copper deficiency in humans. Semin Hematol 20: 118-128, 1983.

29. Ruocco L, Baldi A, Cecconi N, et al. Severe pancytopenia due to copper deficiency. Case report. Acta Haematol 76: 224-226, 1986.

30. Buchman AL, Keen CL, Vinters HV, et al. Copper deficiency secondary to a copper transport defect: a new copper metabolic disturbance. Metabolism 43: 1462-1469, 1994.

31. Wapnir RA, Devas G. Copper deficiency: interaction with highfructose and high-fat diets in rats. Am J Clin Nutr 61: 105-110, 1995.

32. Percival SS. Neutropenia caused by copper deficiency: possible mechanisms of action. Nutr Rev 53: 59-66, 1995.

33. Jayakumar S, Micallef-Eynaud PD, Lyon TD, Cramb R, Jilaihawi AN, Prakash D. Acquired copper deficiency following prolonged jejunostomy feeds. Ann Clin Biochem 42 (Pt 3): 227-231, 2005.

34. Arredondo M, Nunez MT. Iron and copper metabolism. Mol Aspects Med 26: 313-327, 2005.

(C) 2006 The Japanese Society of Internal Medicine http://www.naika.or.jp/imindex.html 